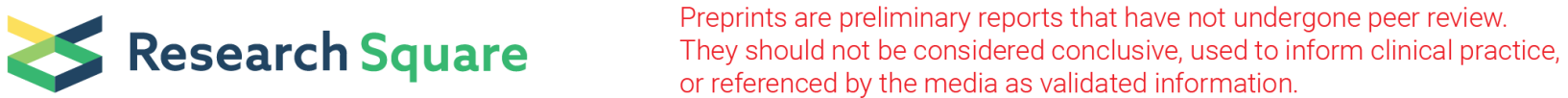

\section{Ovarian metastasis from non-gynecologic primary sites: a retrospective analysis of 177 cases and 13-year experience}

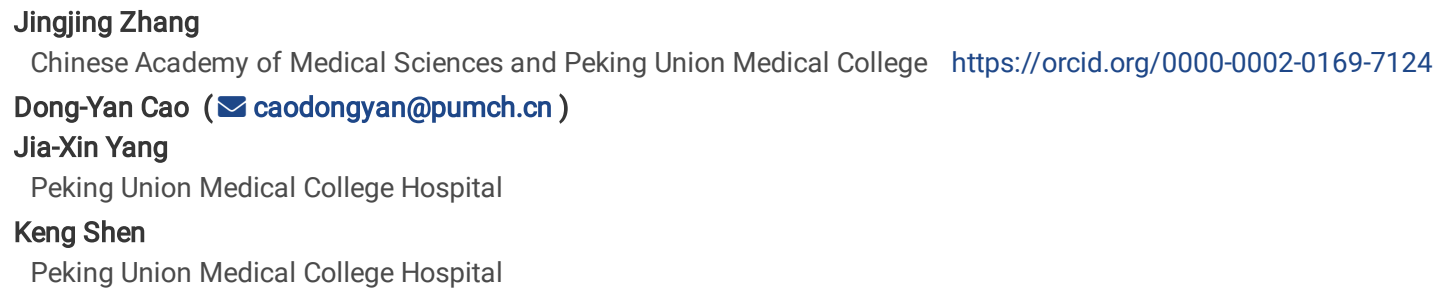

Research

Keywords: Ovarian metastasis, non-gynecologic primary site, cytoreductive surgery, residual lesion, differentiation, prognosis

Posted Date: February 12th, 2020

DOI: https://doi.org/10.21203/rs.2.23362/v1

License: (c) (i) This work is licensed under a Creative Commons Attribution 4.0 International License. Read Full License 


\section{Abstract}

Background

Metastasis to the ovary is not rare. Generally, the most common non-gynecologic primary site of Ovarian metastasis is gastrointestinal tract. Differential diagnosis between primary malignant ovarian tumor and metastatic ovarian tumor is important, otherwise appropriate treatment cannot be determined. Furthermore, optimal treatment strategy for ovarian metastasis from non-gynecologic primary sites still are needed to explore. This study described the clinicopathologic characteristics of ovarian metastasis from non-gynecologic primary sites and identified the significance of surgical treatment for these patients.

Methods

One hundred and seventy-seven patients with ovarian metastasis from non-gynecologic primary sites admitted in Peking Union Medical College Hospital between May 2005 and May 2018 were retrospectively evaluated.

Results

The mean age was 48 years (range 18 - 83). Approximately $60 \%$ of patients were premenopausal women. The mostly two common non-gynecologic primary sites of ovarian metastasis were colorectum (68 cases) and stomach (61 cases). Besides the most common symptoms of abdominal distension (39.0\%), abdominal pain (37.9\%), ascites (27.7\%), there were $18.1 \%$ of patients presenting with abnormal uterine bleeding. A half of patients who tested serum CA- 125 preoperatively had an elevated CA-125 within the range of $35 \mathrm{U} / \mathrm{ml}$ to $200 \mathrm{U} / \mathrm{ml}$. More than $70 \%$ of synchronous ovarian metastasis were misdiagnosed as primary ovarian cancer preoperatively. Of patients, $56.5 \%$ achieved optimal cytoreductive surgery (the diameter of largest residual lesion $<2 \mathrm{~cm}$ ). The overall 5 year survival rate and median survival time were $10 \%$ and 20 months, respectively. The primary sites, optimal cytoreductive surgery, differentiation of tumor, and postoperative adjuvant treatment were prognostic indicators.

Conclusions

Colorectum and stomach are the most common non-gynecologic primary sites of ovarian metastasis. Synchronous ovarian metastasis is easy to be misdiagnosed as primary ovarian cancer. Optimal cytoreductive surgery and postoperative adjuvant treatment can be done to confer survival benefit in selected patients.

\section{Background}

As lung and liver, ovary is a frequent site of metastasis for genital and non-gynecologic primary malignancies[1-3]. Metastasis to the ovary from nongynecologic organs accounts for $9 \%$ of all ovarian malignancies[4]. Gastrointestinal tract is the most common non-gynecologic primary site metastatic to the ovaries, followed by breast[1, 2]

Inappropriate treatment and adverse outcome may occur when ovarian metastasis and primary ovarian cancer are misdiagnosed as each other[5]. An optimum surgical debulking is the most important treatment strategy for ovarian primary cancer because its minimum residual lesion after surgery is associated with a longer term of survival[6]. However, optimal treatment strategy for ovarian metastasis from non-gynecologic primary sites has not been established. First Akhan, S. E. et al. [7] suggested that aggressive surgery should be avoided in patients with extragenital metastatic cancers of the ovary, especially in these complicated with peritoneal metastasis. Because the presence of distant metastasis sites, of course including ovary, usually indicates a dismal prognosis. Then Goere, D. et al. [8] found that ovarian metastasis was less responsive to chemotherapy, so they proposed that ovarian metastasectomy should always be considered, even in the case with non-gynecologic metastases.

The purpose of our study was to evaluate the demographic characteristics, disease presentation, role of surgery and postoperative adjuvant treatment, survival and its prognostic indicators.

\section{Methods}

\section{Study population}

One hundred and seventy-seven patients with ovarian metastasis from non-gynecologic primary sites underwent surgery at Peking Union Medical College Hospital (PUMCH) between May 2005 and May 2018. Clinicopathologic information was obtained from medical records and pathology reports. Follow-up information was obtained from telephone interview. Survival was defined as the date of resection of ovarian metastasis to the date of death or to May 31, 2018. The following data were collected, including: (1) the general conditions; (2) presenting symptoms of ovarian masses related, serum carbohydrate antigen-125(CA-125), preoperative imaging examination; (3) the primary sites, the date of diagnosis of primary cancer, the date of resection of ovarian metastasis, and the interval between the two dates; (4) preoperative diagnosis of ovarian mass; (5) surgical findings, residual lesions size at the completion of surgery, surgical complications; (6) postoperative pathology reports; (7) whether underwent postoperative adjuvant treatment or not; (8) survival time. Ethics approval was granted by the Institutional Review Board of PUMCH.

The chronological sequence of diagnosis was identified as synchronous or metachronous according to the diagnostic date of primary cancer and ovarian metastasis. Namely, the patients with a previous history of non-gynecologic primary cancer before detection of the ovarian metastasis were assigned to the metachronous group; otherwise, they were assigned to the synchronous group. The interval between the two dates in metachronous group is calculated. 
All the enrolled patients underwent surgery in our hospital. According to the surgical findings, extraovarian involvement was determined as positive or negative. In consideration of no uniform definition of radical or palliative surgery, and the diverse surgical removal extent, we focus on the residual lesion size at the completion of surgery. Surgery was considered as optimal if the diameter of the largest residual lesion was less than $2 \mathrm{~cm}$.

All ovarian masses had pathological confirmation of the surgical specimens as ovarian metastasis. According to the pathological results, the degree of differentiation was determined to be well differentiated, moderately differentiated, poorly differentiated and undifferentiated. A majority of primary sites were determined by the previous surgical pathology at our hospital or other hospitals. The rest of the enrolled patients had neither pathology or diagnostic imaging examination to identify the primary sites. These were classified as the group of unknown primary site.

\section{Statistical analysis}

The data were analyzed using SPSS for IOS, version 21. Survival rates were calculated using the life table method, and differences between groups were calculated using the log-rank test. A two-sided $\mathrm{P}$ values of 0.05 or less were regarded as statistically significant.

\section{Results}

\section{Patient characteristics, presenting symptoms, preoperative examinations}

Between May 2005 and May 2018, 177 female patients underwent surgery for ovarian metastasis from non-gynecologic primary sites at our center. The mean age was $47.6 \pm 13.2$ years (range 18-83). Of patients, $59.3 \%$ were premenopausal women. The most common presenting symptoms were abdominal pain (67/177, 37.9\%), abdominal distension (69/177, 39\%), ascites (49/177, 27.7\%), and abnormal uterine bleeding (32/177, 18.1\%). (Table 1)

The preoperative CA-125 value was tested in 116 patients. Serum CA-125 was elevated $(>35 \mathrm{U} / \mathrm{ml})$ in $65.5 \%$ of these patients. Of patients with elevated CA125 , the majority of patients $(77 / 116,66.4 \%)$ had a slight increase of CA-125 $(<200 \mathrm{U} / \mathrm{ml})$. (Table 1$)$

Ovarian mass was found in $98.3 \%$ of the patients by preoperative imaging examination. (Table 1)

Table 1 Patient characteristics of the 177 patients.

\begin{tabular}{|ll|}
\hline Characteristics & N(\%) \\
\hline Age(mean \pm SD, years) & $47.6 \pm 13.2$ \\
\hline Menopausal status & \\
\hline Premenopausal & $105(59.3 \%)$ \\
\hline Postmenopausal & $72(40.7 \%)$ \\
\hline Presenting symptoms & \\
\hline Abdominal pain & $67(37.9 \%)$ \\
\hline Abdominal distension & $69(39.0 \%)$ \\
\hline Ascites & $49(27.7 \%)$ \\
\hline Abnormal uterine bleeding & $32(18.1 \%)$ \\
\hline Preoperative serum CA-125 & \\
\hline Not done & $23(13.0 \%)$ \\
\hline Normal & $38(21.5 \%)$ \\
\hline Abnormal (<200 U/ml) & $77(43.5 \%)$ \\
\hline Abnormal ( $\geq 200$ U/ml) & $39(22.0 \%)$ \\
\hline Preoperative imaging examination & \\
\hline Positive & $174(98.3 \%)$ \\
\hline Negative & $3(1.7 \%)$ \\
\hline
\end{tabular}

\section{Preoperative Diagnosis And Postoperative Pathology}

In our patients, primary cancers were colorectum (68/177, 38.4\%), stomach (61/177, 34.5\%), <link rid="Sec10">appendix</link> (12/177, 6.8\%), biliary tract (7/177, $4.0 \%)$, pancreas $(7 / 177,4.0 \%)$, breast $(6 / 177,3.4 \%)$, small intestine $(5 / 177,2.8 \%)$, lung $(3 / 177,1.7 \%)$, bladder $(2 / 177,1.1 \%)$, unknown sites $(6 / 177$, 3.4\%). (Table 2) 
Table 2

Interval of metachronous ovarian metastasis and differentiation of tumor of the 177 patients.

\begin{tabular}{|c|c|c|c|c|c|c|c|c|c|c|}
\hline & $\begin{array}{l}\text { Colorectum } \\
(n=68)\end{array}$ & $\begin{array}{l}\text { Stomach } \\
(n=61)\end{array}$ & $\begin{array}{l}\text { Biliary } \\
\text { Tract } \\
(n=7)\end{array}$ & $\begin{array}{l}\text { Pancreas } \\
(n=7)\end{array}$ & $\begin{array}{l}\text { Breast } \\
(n=6)\end{array}$ & $\begin{array}{l}\text { Small } \\
\text { intestine } \\
(n=5)\end{array}$ & $\begin{array}{l}\text { Lung } \\
(n=3)\end{array}$ & $\begin{array}{l}\text { Bladder } \\
(n=2)\end{array}$ & $\begin{array}{l}\text { Unknowna } \\
(n=6)\end{array}$ & \\
\hline $\begin{array}{l}\text { Chronological } \\
\text { sequence } \\
\text { Synchronous }\end{array}$ & $40(58.8 \%)$ & $30(49.2 \%)$ & $10(83.3 \%)$ & $2(28.6 \%)$ & $4(57.1 \%)$ & - & $2(40.0 \%)$ & $1(33.3 \%)$ & $1(50.0 \%)$ & $6(100.0 \%)$ \\
\hline Metachronous & $28(41.2 \%)$ & $31(50.8 \%)$ & $2(16.7 \%)$ & $5(71.4 \%)$ & $3(42.9 \%)$ & $6(100.0 \%)$ & $3(60.0 \%)$ & $2(66.7 \%)$ & $1(50.0 \%)$ & - \\
\hline $\begin{array}{l}\text { Median interval } \\
\text { (range, months) }\end{array}$ & $12.5(2-40)$ & $\begin{array}{l}20(1.5- \\
240)\end{array}$ & 95,95 & $\begin{array}{l}51(12- \\
73)\end{array}$ & $\begin{array}{l}\text { 112(19- } \\
115)\end{array}$ & $54(7-81)$ & $\begin{array}{l}18(12- \\
34)\end{array}$ & 4,48 & 135 & - \\
\hline $\begin{array}{l}\text { Differentiation } \\
\text { Well }\end{array}$ & $10(14.7 \%)$ & - & $4(33.3 \%)$ & - & $3(42.9 \%)$ & - & - & - & - & - \\
\hline Moderate & $42(61.8 \%)$ & $6(9.8 \%)$ & $4(33.3 \%)$ & $3(42.9 \%)$ & $2(28.6 \%)$ & - & $2(40.0 \%)$ & - & $1(50.0 \%)$ & $2(33.3 \%)$ \\
\hline Poor/undifferentiated & $16(23.5 \%)$ & $52(85.2 \%)$ & $4(33.3 \%)$ & $1(14.3 \%)$ & $1(14.3 \%)$ & $5(83.3 \%)$ & $3(60.0 \%)$ & $3(100.0 \%)$ & $0(0 \%)$ & $2(33.3 \%)$ \\
\hline Not available & $0(0 \%)$ & $3(4.9 \%)$ & - & $3(42.9 \%)$ & $1(14.3 \%)$ & $1(16.7 \%)$ & - & - & $1(50.0 \%)$ & $2(33.3 \%)$ \\
\hline
\end{tabular}

a. The patients had neither pathology or diagnostic imaging examination to identify the primary cancers who were classified as the group of unknown primary sites.

b. If the number of cases is less than 3 , the intervals are listed.

Ninety-six patients (54.2\%) presented with synchronous ovarian metastasis, while 81 (45.8\%) developed metachronous ovarian metastasis after the diagnosis of primary cancers. In metachronous cases, the median interval was 19 months (range 1.5-240 months). (Table 2)

The differentiation of tumor can be identified in 166 patients. Of them, patients with well differentiation accounted for $9.6 \%$, moderate differentiation for $35 \%$, low differentiation for $49.2 \%$ and undifferentiation for $6.2 \%$. (Table 2 )

According to the clinical manifestations and previous history, a total of 103 patients (58.2\%) were tentatively diagnosed with ovarian metastatic tumor before surgery. Thereinto, 26 patients came from metachronous group and 77 patients came from synchronous group. There was significant difference between the two groups in preoperative diagnostic accuracy $(\mathrm{P}<0.0001)$. (Table 3$)$

Table 3 Preoperative diagnostic accuracy in metachronous and synchronous group.

\begin{tabular}{|c|c|c|c|c|}
\hline & \multicolumn{2}{|c|}{ Tentatively diagnosed with ovarian metastasis } & \multirow[t]{2}{*}{ Diagnostic accuracy } & \multirow[t]{2}{*}{ P-value } \\
\hline & Yes & No & & \\
\hline Synchronous group & 26 & 70 & $27.1 \%$ & \multirow[t]{11}{*}{$<0.0001$} \\
\hline Colorectum & 14 & 26 & & \\
\hline Stomach & 8 & 22 & & \\
\hline 0 & 10 & & & \\
\hline Small intestine & 0 & 2 & & \\
\hline Biliary Tract & 0 & 2 & & \\
\hline Lung & 0 & 1 & & \\
\hline Pancreas & 3 & 1 & & \\
\hline Bladder & 0 & 1 & & \\
\hline Unknown & 1 & 5 & & \\
\hline Metachronous group & 77 & 4 & $95.1 \%$ & \\
\hline
\end{tabular}

\section{Surgery And Postoperative Adjuvant Treatment}

According to the surgical findings, $54.8 \%$ of patients had bilateral ovarian involvement, and $61.6 \%$ of patients had extraovarian involvement. At the completion of surgery, the largest residual lesion less than $2 \mathrm{~cm}$ was achieved in 100 cases (56.5\%). (Table 4)

Surgical complications occurred in approximately $10 \%$ of patients (17/177), including anemia, infection, intestinal obstruction, poor wound healing, cardiovascular-related adverse event. In which, one patient died of pulmonary embolism. (Table 4) 
After surgery, 112 (63.3\%) patients received adjuvant chemotherapy and/or radiation based on the primary cancers based on the primary site. (Table 4)

Table 4 Surgery and postoperative adjuvant therapy of the 177 patients.

\begin{tabular}{|ll|}
\hline & $\mathbf{N}(\%)$ \\
\hline Bilaterality & \\
\hline Bilateral & $97(54.8 \%)$ \\
\hline Unilateral & $80(45.2 \%)$ \\
\hline Median tumor diameter(range, cm) & $8.5(1.0-35.0)$ \\
\hline Extraovarian involvement & \\
\hline Positive & $109(61.6 \%)$ \\
\hline Negative & $68(38.4 \%)$ \\
\hline Residual lesion & \\
\hline$<2$ cm & $100(56.5 \%)$ \\
\hline$\geq 2$ cm & $77(43.5 \%)$ \\
\hline Surgical complications & \\
\hline Yes & $17(9.6 \%)$ \\
\hline No & $160(90.4 \%)$ \\
\hline Adjuvant treatment & \\
\hline Yes & $112(63.3 \%)$ \\
\hline No & $65(36.7 \%)$ \\
\hline
\end{tabular}

\section{Survival}

The overall 3-year survival rate and 5-year survival rate were $23 \%$ and $10 \%$, respectively, and the median survival time was 20 months (95\% Cl: $16-24$ months). (Fig. 1)

There is a significant difference in median survival time between different primary sites $(P=0.02)$. The median survival time of every primary site was as follows: small intestine 59 months, <link rid="Sec10">appendix</link> 54 months, bladder 27 months, breast 25 months, lung 25 months, colorectum 21 months, stomach 18 months, biliary tract 14 months, pancreas 13 months. The median survival times according to the differentiation of tumor showed significant differences $(P=0.016)$ and were as follows: well differentiation 34 months, moderate differentiation 21 months, low differentiation or undifferentiation 16 months (Fig. 2). The median survival time was 25 months in patients whose largest residual lesion was less than $2 \mathrm{~cm}$ and 14 months in those whose largest residual lesion more than or equal to $2 \mathrm{~cm}(P=0.001)$ (Fig. 3). According to the adjuvant treatment there was a significant difference in survival between patients with postoperative chemotherapy and/or radiation and those without postoperative treatment, with an estimated median survival of 24 months and 8 months, respectively $(P<0.001)$ (Fig. 4). However, age, menopausal status, present of ascites, CA-125level $(<200 \mathrm{U} / \mathrm{ml} \mathrm{vs}$. $\geq 200 \mathrm{U} / \mathrm{ml})$, bilaterality of ovarian metastasis, extraovarian involvement, chronological sequence of diagnosis (synchronous vs. metachronous) did not affect survival.

\section{Discussion}

The colorectum $(68 / 177,38.4 \%)$ and stomach $(61 / 177,34.5 \%)$, both belonging to the gastrointestinal tract, were the first two common non-gynecologic primary sites of ovarian metastasis in the current study. Generally, it is consistent with the study of Kim, W. Y. et al.[9] coming from Korea. Their study showed that the primary site was mostly stomach $(73 / 158,46.2 \%)$ and colon $(61 / 158,38.6 \%)$. And another study of Yada-Hashimoto, N. et al. [1] coming from Japan showed that stomach $(15 / 38,39.5 \%)$, breast $(9 / 38,23.7 \%)$, colon $(7 / 38,18.4 \%)$ were the top three primary sites of non-gynecologic organ. Obviously, because gastric cancer is more common in East Asian populations, the stomach is the non-gynecologic primary site of ovarian metastasis. Outside this region, the most common non-gynecologic primary site of ovarian metastasis may be different. A related study from Turkey shows that breast (35/154, 22.7\%) and stomach $(35 / 154,22.7 \%)$ tied for first place[4]. And two American series reported that breast occupied the first place[2, 10]. In our study, the following primary sites were <link rid="Sec10">appendix</link>, biliary tract, pancreas, breast, small intestine, lung, and bladder. According the other published studies, renal pelvis[10], lymph system[1, 4], melanoma of the skin[2], mesothelioma[4, 10], thyroid carcinoma[11] can also be the non-gynecologic primary sites of ovarian metastasis. Generally, a variety of non-gynecologic organs can serve as the primary sites of ovarian metastasis, of which the digestive tract is the most common. This is consistent with our results. An awareness on the primary sites of ovarian metastasis help clinicians to differentiate the primary and metastatic ovarian cancer as well as search a possible primary site purposefully.

The mean age of our patients was 48 years when they were diagnosed; approximately 10 to 15 years younger than the age of primary ovarian cancer (late $50 \mathrm{~s}$ to early $60 \mathrm{~s}$ )[6]. Therefore, this also led that the $59.3 \%$ of our patients was premenopausal women. It is precisely because of the higher blood flow to ovary of premenopausal women that ovarian metastasis occurs[12].

Page 5/13 
In our study, the top three symptoms were abdominal distension (39.0\%), abdominal pain (37.9\%), ascites (27.7\%). These atypical symptoms are similar with the presenting symptoms reported by the study of Ayhan, A. et al. and the study of Kim, W. Y. et al., including distension/pressure symptom,

palpable/abdominopelvic mass, abdominal pain; in addition, a small group of patients in their studies was asymptomatic[4, 9]. One reason for the difficulty in differentiation of primary and metastatic ovarian cancer is that their presenting symptoms can overlap. For primary ovarian tumors, present with 3 to 4 months of abdominal distension or pain is typical[6]. Furthermore, abnormal uterine bleeding occurred in 18.1\% of our patients. LI-CHUN LU et al. [13] reported that $14.3 \%$ of their patients with Krukenberg tumors occurred menstrual irregularity. One study mentioned above showed that only $5.2 \%$ their patients presented abnormal uterine bleeding[4]. Besides, there is a case report on postmenopausal vaginal bleeding as initial presentation of Krukenberg tumor[14]. Abnormal uterine bleeding is considered to be caused by the ovarian metastasis disrupting ovarian function and affecting sex hormone levels.

Among our patients who tested serum CA-125 before surgery, approximately a half of the patients had an abnormally elevated CA-125 of more than $35 \mathrm{U} / \mathrm{ml}$ but no more than $200 \mathrm{U} / \mathrm{ml}$. Serum CA-125 is the most common tumor marker for ovarian cancer with a low specificity and sensitivity[15]. Although recent work has suggested the change of CA-125 levels over time is more useful in identifying and monitoring ovarian cancer, but the absolute level of CA-125 may help to differentiate primary and metastatic ovarian cancer. Clinically, epithelial ovarian cancer with CA125 up to several thousand is relatively common, while metastatic ovarian cancer is not. Although many tumor markers of gastrointestinal cancer, such as CA-19-9, CEA, CA-72-4, are also with a low sensitivity and specificity $[16,17]$, these elevated tumor markers could serve as a hint for clinicians preoperatively. Additionally, tumor markers with differential diagnostic value include CA199, which is often elevated in pancreatic cancer, and CA153, which is often elevated in breast cancer. But, unfortunately, these tumor markers were not tested as common as CA-125 in patients with ovarian masses.

Patients of metachronous group accounted for $45.8 \%$ in our study. They had a primary cancer in the past and then developed ovarian metastasis with a median interval of 19 months (range 1.5-240 months). Different primary tumors showed a lot of variation in interval: ovarian metastasis from bladder cancer, pancreatic cancer and <link rid="Sec10">appendix</link> cancer often occur in approximately 10 years after the primary tumors; biliary tract cancer, breast cancer and lung cancer in 4 to 5 years; stomach cancer, small intestine cancer, colorectal cancer in 1 to 2 years. Kim, W. Y. et al. [9] showed that their stomach cancer patients and colon cancer patients developed ovarian metastasis in median time of 15.5 months and 13.5 months, respectively; their three breast cancer patients in 41, 47, 73 months. Taranto, A. J. et al. [18] reported a case of ovarian metastasis arising from gall bladder carcinoma with a diagnosis of 4 years previously. And two cases of non-functional neuroendocrine carcinoma of the pancreas developed ovarian metastasis 5 and 7 years after diagnosis, respectively[19, 20]. These findings are nearly consistent with ours. However, the reported several non-small lung cancer patients showed an interval of 14 to 20 months[21, 22] which seems shorter than ours. Considering that this may be related to the pathological type of lung cancer, we further confirmed that the pathological type of our enrolled lung cancer patients was all small-cell lung cancer. Generally, the non-small lung cancer is with a higher malignancy degree and poorer prognosis than small lung cancer. So that explains why our metachronous lung cancer patients were with a longer interval. In addition, out of the primary sites of our study, Corrado, G. et al.[11] reported a rare case of ovarian metastasis from thyroid carcinoma after 9 years from the diagnosis. Over time after diagnosis of non-gynecologic primary cancer, clinicians have better to keep an improved consciousness in development of ovarian metastasis. It is valuable not just because ovarian metastatic tumor cannot be quite uncommon, but because that familiarity with the probable intervals for different primary cancers is helpful for clinicians to tentatively diagnose ovarian metastasis.

Indeed, $58.2 \%$ of our patients were tentatively diagnosed with ovarian metastasis before surgery in which almost all were patients of metachronous group. The preoperative diagnostic accuracy is $95.1 \%$ and $27.1 \%$ in metachronous and synchronous group, respectively. A significant lower accuracy in synchronous group indicates that synchronous ovarian metastasis misdiagnosed as primary ovarian cancer is quite common. Patients with synchronous ovarian metastasis from <link rid="Sec10">appendix</link>, small intestine, biliary tract, lung, and bladder were all diagnosed before surgery incorrectly. Of synchronous ovarian metastasis, $65 \%$ from colorectum and $73.3 \%$ from stomach were misdiagnosed as primary ovarian cancer. Furthermore, of particular note is no breast cancer patients occurred synchronous ovarian metastasis in our study, which may be related to early detection of breast cancer. Unfortunately, little research has been conducted in analyzing synchronous ovarian metastasis mimicked by primary ovarian cancer. Taking no account of the chronological sequence of diagnosis, based on the data available in the literature, $32 \%$ of mimicking metastasis were from colorectum and $49 \%$ of these were from gastrointestinal tract[23,24]. Even though differentiation of synchronous ovarian metastasis and primary ovarian cancer is a difficult problem, yet to be resolved. With possibility of ovarian metastasis in mind, patients presenting ovarian masses of unknown origin should undergo an extensive search to rule out the suspicious primary sites by a full usage of various imaging examinations, gastrointestinal endoscopy, serum CA-125 and other biomarkers and so on. But if these methods cannot help to confirm diagnosis, surgical resection and pathology of the ovary should a last resort.

The purpose of surgical intervention is not only to establish a definite diagnosis, but also to alleviate symptoms, minimize residual tumor burden, avoid progression or achieve a cure. Usually, surgeons proceed with surgical intervention depending on the presence of severe symptoms or emergencies, or surgical removal of tumors deemed necessary for asymptomatic patients following a multidisciplinary team consensus. Due to the variety of the tumor size, the depth of invasion, involvement, the surgical removal extent was various in which the minimal one was only unilateral salpingo-oophorectomy and the maximal one can include resection of multiple involved organs and local lymph nodes. Over the past decade, an amount of retrospective studies has proven that the residual lesion size at the completion of surgery is related to a survival benefit for patients with ovarian metastasis from non-gynecologic primary cancers[1, 4 , 9, 25-32]. In our current study, there was a significant survival difference between patients with less than patients with $2 \mathrm{~cm}$ of largest residual lesion and more than or equal to $2 \mathrm{~cm}$ of largest residual lesion. Median survival time of the two groups were 25 months and 14 months, respectively. The result might indicate us every effort to perform optimal surgery should be made. However, resectability of tumors and underlying surgical complications were required to evaluate before surgery. Surgeons made the decision to proceed with surgery after balancing surgical benefits and risks. In our series, although approximately $10 \%$ of patients experienced complications, the fatal complication was only observed in one patient, which is consistent with Seow-En, I.'s results[33]. Generally speaking, surgery for patients of ovarian metastasis from non-gynecologic primary cancers can be performed safely with an acceptable complication rate. 
Prognosis of patients with ovarian metastasis from non-gynecologic primary sites is poor. The published study of the largest sample size (158 patients) reported that the 5 -year survival rate and median survival time were $7.2 \%$ and 15 months, respectively[9]. These results were not different from ours. In our study, the 3-year survival rate, 5-year survival rate, and median survival time were $23 \%, 10 \%$, and 20 months, respectively. Besides the residual lesion size, our results showed that primary sites, differentiation of ovarian metastasis and postoperative adjuvant treatment were also prognostic indicators.

A considerable amount of studies has reported that survival of patients with ovarian metastasis according to the primary tumor showed significant differences[1, 2, 4, 7, 9, 25, 29, 31,34]. Although the primary sites included in each study were different, among the included common non-gynecologic primary cancers, the overall survival time of breast cancer was longer than that of gastrointestinal cancer, and that of colorectal cancer was longer than that of gastric cancer. In our study, the median survival time of breast cancer, colorectal cancer and stomach cancer were 25 months, 21 months and 18 months, respectively; consistent with the results of aforementioned earlier studies. Moreover, for the reason of a more variety of non-gynecologic primary sites included in our study than other studies, some cancers with relatively good prognosis, like <link rid="Sec10">appendix</link> mucinous adenocarcinoma and bladder transitional cell carcinoma, survive longer after resection of ovarian metastasis, whose median survival time were 54 months and 27 months, respectively. On the contrary, patients of biliary tract and pancreatic cancers with poorer prognosis died one year after surgery for ovarian metastasis in our study. All the 5 enrolled cases of small intestine cancer were adenocarcinoma. According to the literature, small intestinal adenocarcinoma prognosis seems at an intermediate stage of that of colon and gastric cancers[35]. While the 59 months of median survival time of small intestine cancer after surgery was the longest in our study. This seemingly indicates that prognosis is not entirely consistent with postoperative survival time for ovarian metastasis. Due to the lack of relevant studies, it is not possible to explain this for the time being.

To our knowledge, no study specially analyzed the differentiation of ovarian metastasis as an underlying prognostic indicator. Nevertheless, our results showed that the median survival time of patients with ovarian metastasis well, moderate, low differentiation or undifferentiation were 34 months, 21 months, 16 months, respectively; which was a significant difference.

Postoperative adjuvant treatment is identified as essential. Both an early study[9] and our current studies have proven that the patients who underwent postoperative adjuvant treatment, mainly chemotherapy, survived longer than those who did not.

According to the aforementioned prognostic indicators, patients with ovarian metastasis from non-gynecologic primary sites can be selected to undergo optimal cytoreductive surgery which confers survival benefits with a low rate of surgical complications.

\section{Conclusion}

It is worthy of consideration to perform surgery for patients with ovarian metastasis from non-gynecologic primary sites. The decision to proceed with cytoreductive surgery is affected by presence of symptoms, the primary sites, differentiation of tumor. In an experienced hospital, a multidisciplinary team should evaluate resectability of tumors before surgery. Every effort should be made to achieve the an optimal cytoreductive surgery, namely the diameter of largest residual lesion at the completion of surgery are less than $2 \mathrm{~cm}$, followed by postoperative adjuvant treatment.

\section{Abbreviations}

PUMCH: Peking Union Medical College Hospital; CA-125: carbohydrate antigen-125;CA19-9: cancer antigen 19-9; CA72-4: cancer antigen 72-4; CEA: carcinoembryonic antigen

\section{Declarations}

Ethics approval and consent to participate

Ethics approval was granted by the Institutional Review Board (IRB) of Peking Union Medical College Hospital (PUMCH). The ethical review reference of the study is S-K912.

Consent for publication

Not applicable.

Availability of data and materials

The dataset used or analysed including in this study are available from the co- corresponding authors on reasonable request.

Competing interests

The authors declare that they have no competing interests. 
Funding

Not applicable.

Author Contributions

KS and DY-C developed the idea and designed the study. KS, JX-Y, DY-C and JJ-Z were involved in the diagnosis, treatment and follow-up of the enrolled patients. JJ-Z collected and analysed the data. JJ-Z and DY-C preformed the drafting of the manuscript. All the authors read and approved the final manuscript.

Acknowledgements

We appreciate the stuff at PUMCH for their diligent clinical work and precise data recording about the patients we enrolled in this article.

\section{References}

1. Yada-Hashimoto N, Yamamoto T, Kamiura S, Seino H, Ohira H, Sawai K, Kimura T, Saji F: Metastatic ovarian tumors: a review of 64 cases. Gynecologic oncology 2003, 89(2):314-317.

2. de Waal YR, Thomas CM, Oei AL, Sweep FC, Massuger LF: Secondary ovarian malignancies: frequency, origin, and characteristics. International journal of gynecological cancer : official journal of the International Gynecological Cancer Society 2009, 19(7):1160-1165.

3. Doganay M, Topcu HO, Kokanali MK, Guzel Al, Oskovi A, Akbay S, Cavkaytar S: Krukenberg carcinoma metastasized from stomach resembling mucinous cystadenocarcinoma of the ovary. Journal of experimental therapeutics \& oncology 2015, 11(1):23-26.

4. Ayhan A, Guvenal T, Salman MC, Ozyuncu O, Sakinci M, Basaran M: The role of cytoreductive surgery in nongenital cancers metastatic to the ovaries. Gynecologic oncology 2005, 98(2):235-241.

5. Young RH, Scully RE: Metastatic tumors in the ovary: a problem-oriented approach and review of the recent literature. Seminars in diagnostic pathology 1991, 8(4):250-276.

6. Jayson GC, Kohn EC, Kitchener HC, Ledermann JA: Ovarian cancer. Lancet (London, England) 2014, 384(9951):1376-1388.

7. Akhan SE, Kilic G, Salihoglu Y, Bengisu E, Berkman S: Nongenital metastatic cancers of the ovary: a clinical analysis. European journal of gynaecological oncology 2001, 22(5):379-383.

8. Goere D, Daveau C, Elias D, Boige V, Tomasic G, Bonnet S, Pocard M, Dromain C, Ducreux M, Lasser P et al: The differential response to chemotherapy of ovarian metastases from colorectal carcinoma. European journal of surgical oncology : the journal of the European Society of Surgical Oncology and the British Association of Surgical Oncology 2008, 34(12):1335-1339.

9. Kim WY, Kim TJ, Kim SE, Lee JW, Lee JH, Kim BG, Bae DS: The role of cytoreductive surgery for non-genital tract metastatic tumors to the ovaries. European journal of obstetrics, gynecology, and reproductive biology 2010, 149(1):97-101.

10. Demopoulos RI, Touger L, Dubin N: Secondary ovarian carcinoma: a clinical and pathological evaluation. International journal of gynecological pathology : official journal of the International Society of Gynecological Pathologists 1987, 6(2):166-175.

11. Corrado G, Pomati G, Russo A, Visca P, Vincenzoni C, Patrizi L, Vizza E: Ovarian metastasis from thyroid carcinoma: a case report and literature review. Diagnostic pathology 2014, 9:193.

12. Miller BE, Pittman B, Wan JY, Fleming M: Colon cancer with metastasis to the ovary at time of initial diagnosis. Gynecologic oncology 1997, 66(3):368371.

13. Lu LC, Shao YY, Hsu CH, Hsu C, Cheng WF, Lin YL, Cheng AL, Yeh KH: Metastasectomy of Krukenberg tumors may be associated with survival benefits in patients with metastatic gastric cancer. Anticancer research 2012, 32(8):3397-3401.

14. Kumar M, Kumar A, Maroules M, Abrina V, Kumar V: Postmenopausal vaginal bleeding as initial presentation of gastric cancer: a case report with literature review of prognostic factors and treatment of krukenberg tumor. Annals of translational medicine 2016, 4(4):84.

15. Lowry KP, Lee SI: Imaging and Screening of Ovarian Cancer. Radiologic clinics of North America 2017, 55(6):1251-1259.

16. van der Schouw YT, Verbeek AL, Wobbes T, Segers MF, Thomas CM: Comparison of four serum tumour markers in the diagnosis of colorectal carcinoma. British journal of cancer 1992, 66(1):148-154.

17. Carpelan-Holmstrom M, Louhimo J, Stenman UH, Alfthan H, Haglund C: CEA, CA 19-9 and CA 72-4 improve the diagnostic accuracy in gastrointestinal cancers. Anticancer research 2002, 22(4):2311-2316.

18. Taranto AJ, Lourie R, Lau WF: Ovarian vascular pedicle sign in ovarian metastasis arising from gall bladder carcinoma. Australas Radio/2006, 50(5):504506.

19. La Rosa S, Marando A, Ghezzi F, Colombo P, Finzi G, Capella C: Cushing's syndrome due to a pancreatic neuroendocrine tumor metastatic to the ovaries: a clinicopathological description of a case. Endocrine pathology 2011, 22(2):118-124.

20. Watt DG, Pandanaboyana S, Herrington CS, Tait IS: Pancreatic glucagonoma metastasising to the right ovary five years after initial surgery: a case report. JOP : Journal of the pancreas 2013, 14(5):510-514. 
21. Fujiwara A, Higashiyama M, Kanou T, Tokunaga T, Okami J, Kodama K, Nishino K, Tomita Y, Okamoto I: Bilateral ovarian metastasis of non-small cell lung cancer with ALK rearrangement. Lung Cancer 2014, 83(2):302-304.

22. Mushi RT, Yang Y, Cai Q, Zhang R, Wu G, Dong X: Ovarian metastasis from non-small cell lung cancer with ALK and EGFR mutations: A report of two cases. Oncol Lett 2016, 12(6):4361-4366.

23. Mazur MT, Hsueh S, Gersell DJ: Metastases to the female genital tract. Analysis of 325 cases. Cancer 1984, 53(9):1978-1984.

24. Moore RG, Chung M, Granai CO, Gajewski W, Steinhoff MM: Incidence of metastasis to the ovaries from nongenital tract primary tumors. Gynecologic oncology 2004, 93(1):87-91.

25. Petru E, Pickel H, Heydarfadai M, Lahousen M, Haas J, Schaider H, Tamussino K: Nongenital cancers metastatic to the ovary. Gynecologic oncology 1992, 44(1):83-86.

26. Kim HK, Heo DS, Bang YJ, Kim NK: Prognostic factors of Krukenberg's tumor. Gynecologic oncology 2001, 82(1):105-109.

27. Kobayashi O, Sugiyama Y, Cho H, Tsuburaya A, Sairenji M, Motohashi H, Yoshikawa T: Clinical and pathological study of gastric cancer with ovarian metastasis. International journal of clinical oncology 2003, 8(2):67-71.

28. Cheong JH, Hyung WJ, Chen J, Kim J, Choi SH, Noh SH: Surgical management and outcome of metachronous Krukenberg tumors from gastric cancer. Journal of surgical oncology 2004, 87(1):39-45.

29. Jiang R, Tang J, Cheng X, Zang RY: Surgical treatment for patients with different origins of Krukenberg tumors: outcomes and prognostic factors. European journal of surgical oncology : the journal of the European Society of Surgical Oncology and the British Association of Surgical Oncology 2009, 35(1):92-97.

30. Ulker V, Numanoglu C, Alpay V, Akbayir O, Polat I, Gedikbasi A, Akca A: Characteristics and prognosis of ovarian metastatic tumors: review of a singleinstitution experience. European journal of gynaecological oncology 2013, 34(1):75-78.

31. Wu F, Zhao X, Mi B, Feng LU, Yuan NA, Lei F, Li M, Zhao X: Clinical characteristics and prognostic analysis of Krukenberg tumor. Molecular and clinical oncology 2015, 3(6):1323-1328.

32. Lu W, Yuan L, Liu X, Guo S-W: Identification of prognostic factors for Krukenberg tumor. Gynecology and Minimally Invasive Therapy 2013, 2(2):52-56.

33. Seow-En I, Hwarng G, Tan GHC, Ho LML, Teo MCC: Palliative surgery for Krukenberg tumors - 12-year experience and review of the literature. World journal of clinical oncology 2018, 9(1):13-19.

34. Guzel AB, Gulec UK, Paydas S, Khatib G, Gumurdulu D, Vardar MA, Altintas A: Preoperative evaluation, clinical characteristics, and prognostic factors of nongenital metastatic ovarian tumors: review of 48 patients. European journal of gynaecological oncology 2012, 33(5):493-497.

35. Aparicio T, Zaanan A, Svrcek M, Laurent-Puig P, Carrere N, Manfredi S, Locher C, Afchain P: Small bowel adenocarcinoma: epidemiology, risk factors, diagnosis and treatment. Dig Liver Dis 2014, 46(2):97-104.

\section{Figures}


Survival analysis function

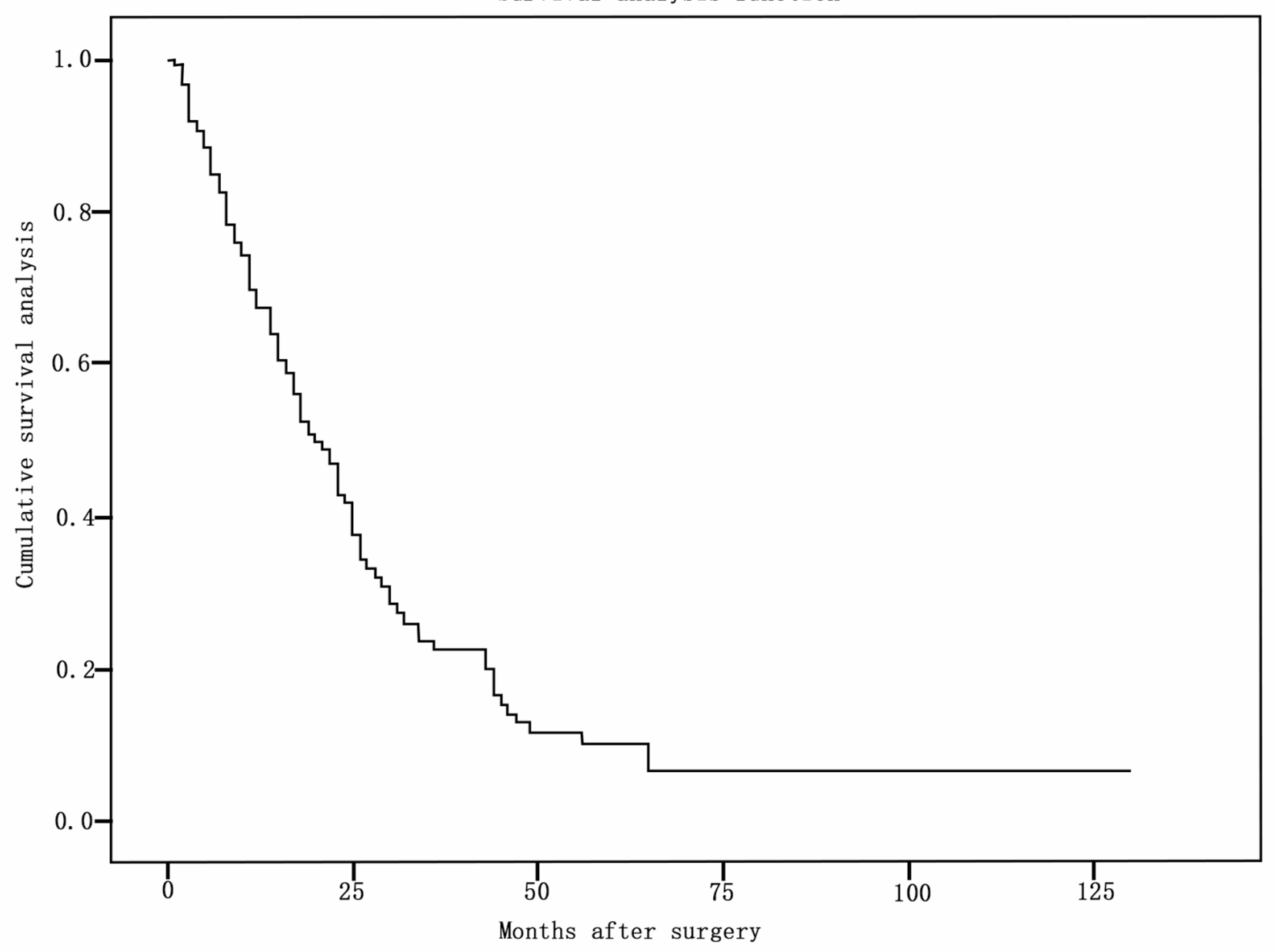

Figure 1

Overall survival of 177 patients. The overall 3-year survival rate and 5-year survival rate were $23 \%$ and $10 \%$, respectively, and the median survival time was 20 months (95\% Cl: 16-24 months). 


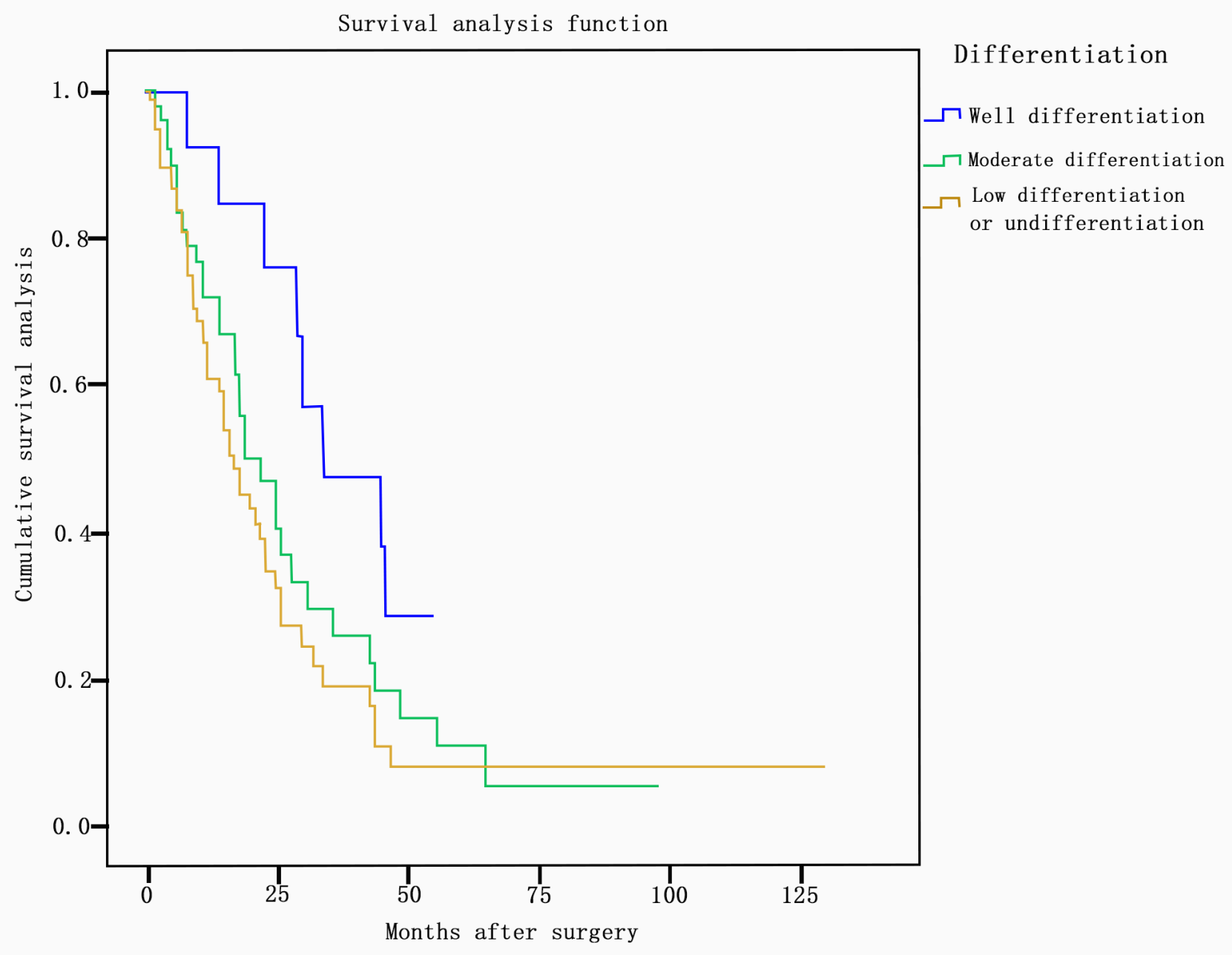

Figure 3

Survival according to differentiation (well differentiation 34 months, moderate differentiation 21 months, low differentiation or undifferentiation 16 months). The median survival times according to the differentiation of tumor showed significant differences $(P=0.016)$. 


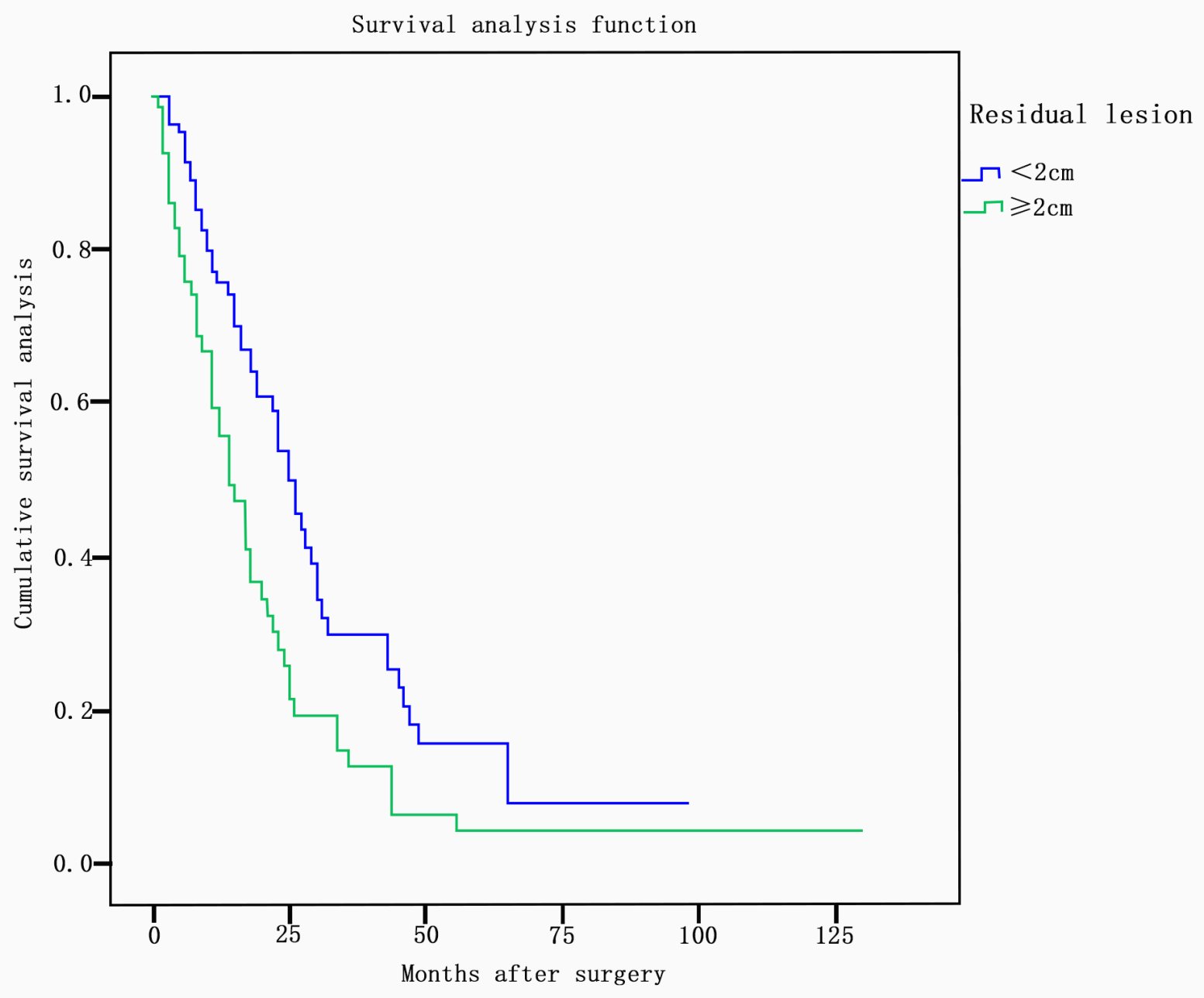

Figure 6

Survival according to residual lesion $(<2 \mathrm{~cm}$ or $\geq 2 \mathrm{~cm}$ ). The median survival time was 25 months in patients whose largest residual lesion was less than 2 $\mathrm{cm}$ and 14 months in those whose largest residual lesion more than or equal to $2 \mathrm{~cm}(P=0.001)$. 
Survival analysis function

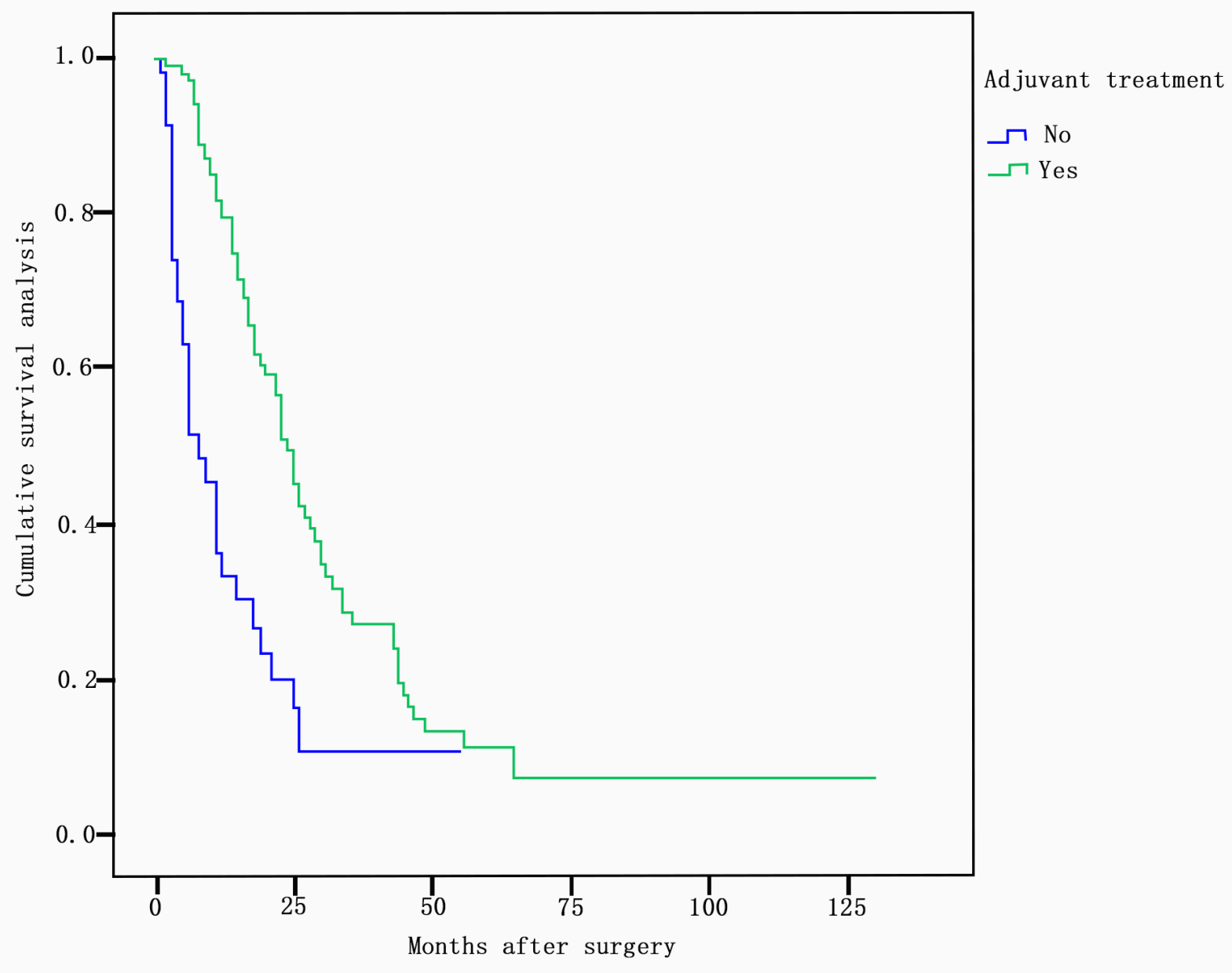

Figure 8

Survival according to postoperative adjuvant treatment. There was a significant difference in survival between patients with postoperative adjuvant treatment and those without postoperative adjuvant treatment, with an estimated median survival of 24 months and 8 months, respectively $(P<0.001)$. 